\title{
Visual Working Efficiency Analysis Method of Cockpit Based On ANN
}

\author{
Yingchun CHEN \\ Commercial Aircraft Corporation of \\ China,Ltd \\ Shanghai, China
}

\author{
Dongdong WEI \\ Fudan University \\ Dept. of Mechanics an \\ Science Engineering \\ Shanghai, China
}

\author{
Gang SUN \\ Fudan University \\ Dept. of Mechanics and \\ Science Engineering \\ Shanghai, China
}

\begin{abstract}
The Artificial Neural Networks method is applied on visual working efficiency of cockpit. A Self-Organizing Map (SOM) network is demonstrated selecting material with near properties. Then a Back-Propagation (BP) network automatically learns the relationship between input and output. After a set of training, the BP network is able to estimate material characteristics using knowledge and criteria learned before. Results indicate that trained network can give effective prediction for material.
\end{abstract}

Keywords- component; Visual Working Efficiency; Artificial Neural Networks;Cockpit; BP; SOM.

\section{INTRODUCTION}

Modern science and technology are people-oriented. Taken more and more human factors into consideration on the development of modern civil airplane, a subject of applying ergonomics into man-machine relationship develops gradually, which has brought about more and more attention.

In the study of man-machine environment system, ergonomics experienced three phases, which were peopleadapted-to-machine, machine-adapted-to-man and manmachine mutual adaptation [1]. Now it has already gone deep into a man-machine environment system of people, machine and environment coordinating with each other. In this system, purely studying on individual physiological and psychological characteristics has been developed into studying on how to improve a person's social factors. With market competition intensified and production level advanced, application of ergonomics in the design and manufacturing of mechanical products also is more wide and deep.

In the man-machine system, the size of each component of human body, the normal physiological values of man's vision and audition, the pose of man in work, human activities range, action rhythm and speed, fatigue degree caused by working conditions, and one's energy consumption and supplement; machine monitor, controller (handle, joysticks, steering wheel, button's structure and tonal, etc.), and various equipment (chair, table, etc.) associated with other people; environment temperature, humidity, noise, vibration, lighting, color, smell, etc will affect one person's working efficiency. Man-machine ergonomics is a subject studying the relationship of them.

Research direction of Man-machine ergonomics mainly displays in the following respects: visual factor (harmonious and pleased environment in both inside and outside cockpit), audition factor (quiet cockpit and cabin), tactile factor (comfortableness of seat and flight equipment), space factor (wild and uncrowded space of cockpit), and the relationship of safety, high efficiency and comfort.

The visual factor plays a very important role among them accounting for the fact that vision is the most important channel communicated with external world for people. About $80 \%$ information received from outside is obtained through the visual pathway. The main interface between man and machine in man-machine system is visual displayer [2]. Results have shown that, warm color causes eyes fatigue easier than cool color. Green and yellow characters cause eyes fatigue lighter than red and blue characters [3]. Green characters cause eyes fatigue smaller than white characters. Besides color, brightness, contrast, and matching of background color, target color also make a different effect to eyes [3].

This paper focused on how different materials affect the cockpit's visual performance in direct sunlight.

\section{ARTIFICIAL NEURAL NETWORK}

Artificial Neural Networks (ANNs) are called Neural Networks (NNs) or Connectionist Model in short [4, 5]. They're a kind of algorithm mathematical model which can simulate animals' behavior characteristics of neural networks and conduct distributed parallel information processing. These networks rely on the complexity of system by adjusting the relationship of the large internal mutual connections of nodes, to process information. Artificial Neural Networks have the capacity of self-learning and self-adaption. Providing a batch of mutual correspondence input/output data in advance, ANNs can analyze the potential law and calculate output with the final new input data according to these laws. These study and analysis process are called 'training'. Characteristics and superiority of ANNs are reflected in three aspects: Firstly, ANNs have function of self-learning. Secondly, ANNs have function of association and storage. Thirdly, ANNs have ability of seeking for optimal solution with high speed. Therefore, ANNs are widely used in medical, automatic control etc, and have important application in dealing with combinatorial optimization problem, pattern recognition and image processing [6].

Self-organization Kohonen network and multilayer perceptron BP network are two artificial neural networks commonly used. The former is mainly used for pattern 
analysis and pattern recognition. The latter is mainly used to approximate complex non-linear relationship of input and output [7][8].

\section{DATABASE PROFILE AND RESEARCH DIRECTION}

As shown in Figure 1, the database is composed by 750 independent data. Each data has information of 12 dimensions beside material ID as shown in Table I.

In the database, obviously, color temperature of light source is $6000 \mathrm{~K}$. Transmissivity of each material is zero (light-proof material). The sum of reflectivity and absorptivity is 100. Therefore, these 3 columns are invalid data which could be rejected. Two relations have been summarized following with reminding data of 9 dimensions.

[brightness, luminous intensity]

$=f[$ material properties, light source properties $]$

[contrast, color coordinate $]=g[$ material properties $]$

TABLE I.

\begin{tabular}{|c|c|c|c|c|c|}
\hline \multicolumn{4}{|c|}{ Input Information } & \multicolumn{2}{|c|}{ Output Information } \\
\hline \multicolumn{2}{|r|}{ Material properties } & \multicolumn{2}{|r|}{ Illuminant properties } & & \\
\hline $\begin{array}{l}\text { Column } \\
\text { Number }\end{array}$ & Name & $\begin{array}{l}\text { Column } \\
\text { Number }\end{array}$ & Name & $\begin{array}{l}\text { Column } \\
\text { Number }\end{array}$ & Name \\
\hline 1 & Reflectivity & 6 & Luminous flux & 8 & Brightness \\
\hline 2 & Transmissivity & 7 & Illuminant Color Temperature & 9 & Luminous Intensity \\
\hline 3 & Absorptivity & & & 10 & Contrast \\
\hline 4 & Material Coordinate $\mathrm{x}$ & & & 11 & Color Coordinate $\mathrm{x}$ \\
\hline 5 & Material Coordinate y & & & 12 & Color Coordinate y \\
\hline
\end{tabular}

Due to the formula (2), contrast and color coordinates of output only relate to material properties (reflectivity, color coordinate $\mathrm{x}$, color coordinate $\mathrm{y}$ ). After contrast and coordinates of output analyzed, materials with draw near properties are chosen for the purpose of further screening. SOM network fits this part of job.

After material finalized, the value of brightness and luminous intensity could be obtained based on approximate input/output relationship by BP network. Thus, the optimal light source condition would be determined. This part of job is completed by BP network.

\section{Simulation RESUlts}

\section{A. Summary of research results based on SOM network}

Contrast and color coordinates only rely on material. Total 15 groups of three dimensional data involving contrast and color coordinates are sampled from 15 kinds of materials accordingly. The distribution of sample points is shown in Figure 2(a).

As shown in Figure 2(a), almost all sample points are ranked in a straight line because each degree of freedom has different scale. This sample will bring adverse impact for SOM network which needs standardization. Standardization uses a square affine transformation with a vertex as original point. Sample points after standardization are shown in Figure 2(b). p.s. The left sides of formula (1) and (2) are output information, while the right sides are input information, as well as symbols of function.

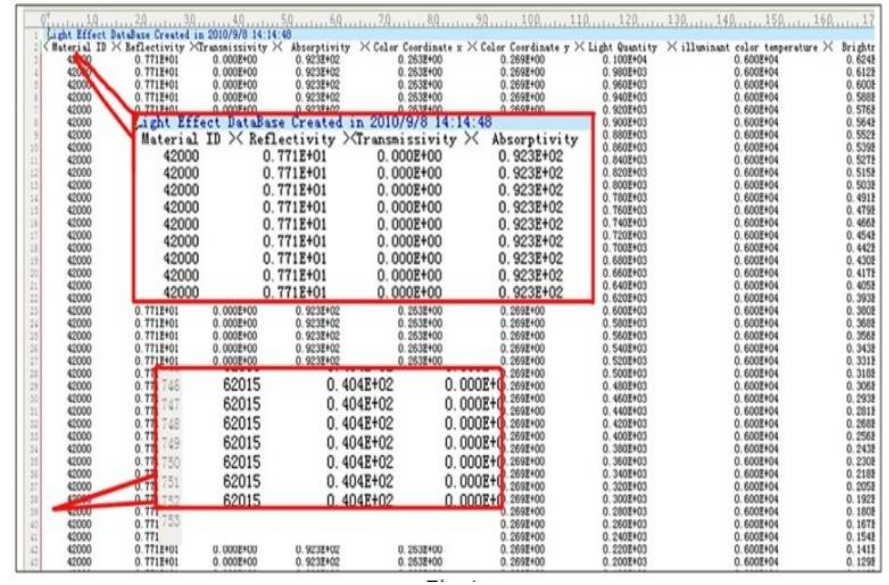

Figure 1. Database Scheme

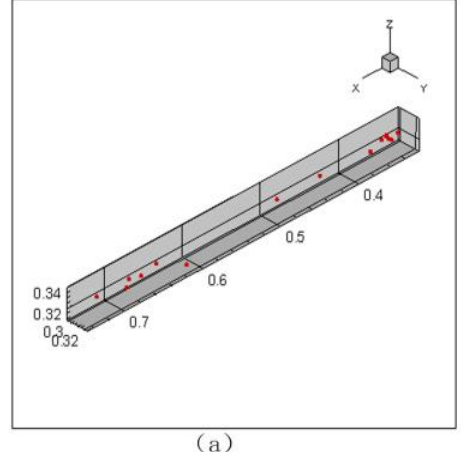

(a)

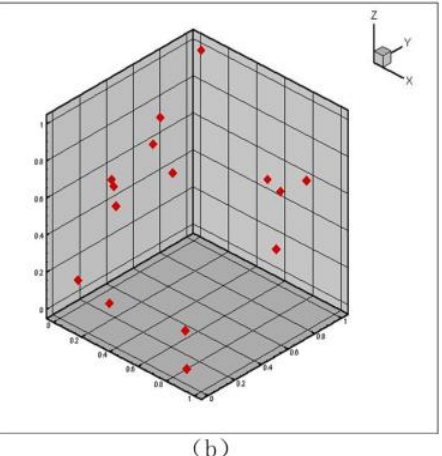

(b)
Figure 2. Distribution of sample points: (a) original distribution of sample points and (b) distribution of sample points after transformation

Initialization parameters of SOM network are shown in Table II. After initializing, parameters of the training process of SOM network have various alternatives. After trying, training results perform well when parameters are set according to Table III.

After training, error curve is plotted and shown in Figure 3, indicating that the error will be less than 10e-4 after 5500 steps. When the training of neural network is completed, response of each neuron is obtained according to every input pattern. Respond surfaces of all samples are shown in Figure 4(a).

TABLE II. 


\begin{tabular}{llll}
\hline $\begin{array}{c}\text { Topological } \\
\text { Structure }\end{array}$ & $\begin{array}{c}\text { Dimension of } \\
\text { SOM network }\end{array}$ & $\begin{array}{c}\text { Function Type of } \\
\text { Epsilon } \\
\text { Neighborhood }\end{array}$ & $\begin{array}{c}\text { Initializing } \\
\text { Pattern }\end{array}$ \\
\hline Rect & $10 * 10$ & Gaussian & random \\
\hline
\end{tabular}

TABLE III.

\begin{tabular}{lllll}
\hline & $\begin{array}{c}\text { Training } \\
\text { Times }\end{array}$ & $\begin{array}{c}\text { Changing Rule } \\
\text { of Learning } \\
\text { Rate }\end{array}$ & $\begin{array}{c}\text { Initial } \\
\text { Learning } \\
\text { Rate }\end{array}$ & $\begin{array}{c}\text { Initial Superior } \\
\text { radius of } \\
\text { neighbourhood }\end{array}$ \\
\hline 1 & 501 & Linear & 0.1 & 10 \\
2 & 5001 & Inverse & 0.03 & 3 \\
\hline
\end{tabular}

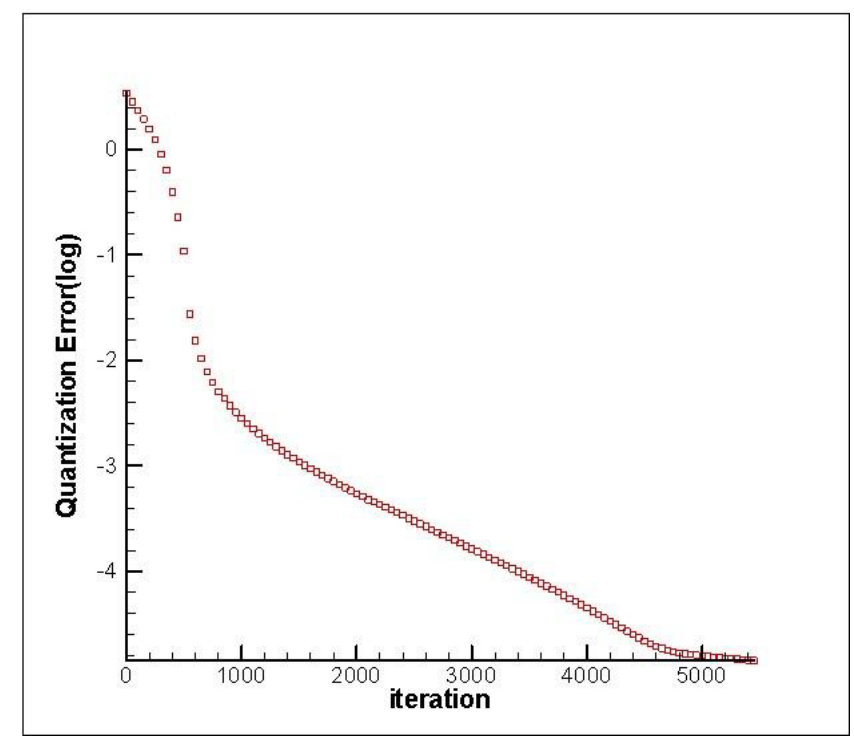

Figure 3. Error curve
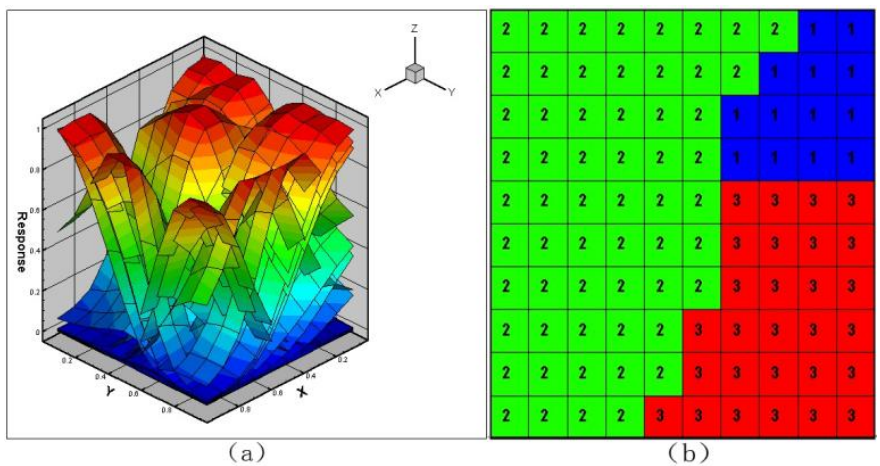

Figure 4. (a) respond surfaces and (b) mapping

All of 15 input samples are divided into groups in according to the above respond surfaces, as shown in Table IV. Samples divided into the same group can active neurons in the same district, which can produce maximum responses. Thus, a 2dimension mapping is shown in Figure 4(b). What is shown in Figure 5 are 2-dimension response diagrams of representative samples of above 3 groups. Scattered sample points are divided into 3 groups by certain rules in Figure 6 .

TABLE IV.

\begin{tabular}{l|l}
\hline Number & Sample Number \\
\hline
\end{tabular}

\begin{tabular}{l|lllllll}
\hline 1 & 1 & 3 & & & & \\
2 & 2 & 4 & 5 & 6 & 8 & 10 & 12 \\
3 & 7 & 9 & 11 & 13 & & & \\
\hline
\end{tabular}

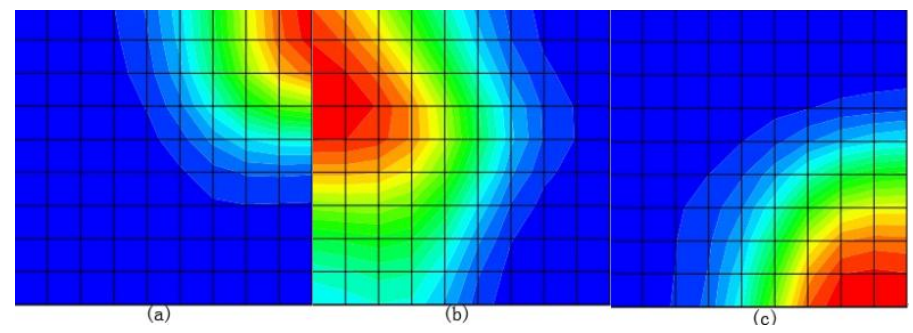

Figure 5. 2d response diagram

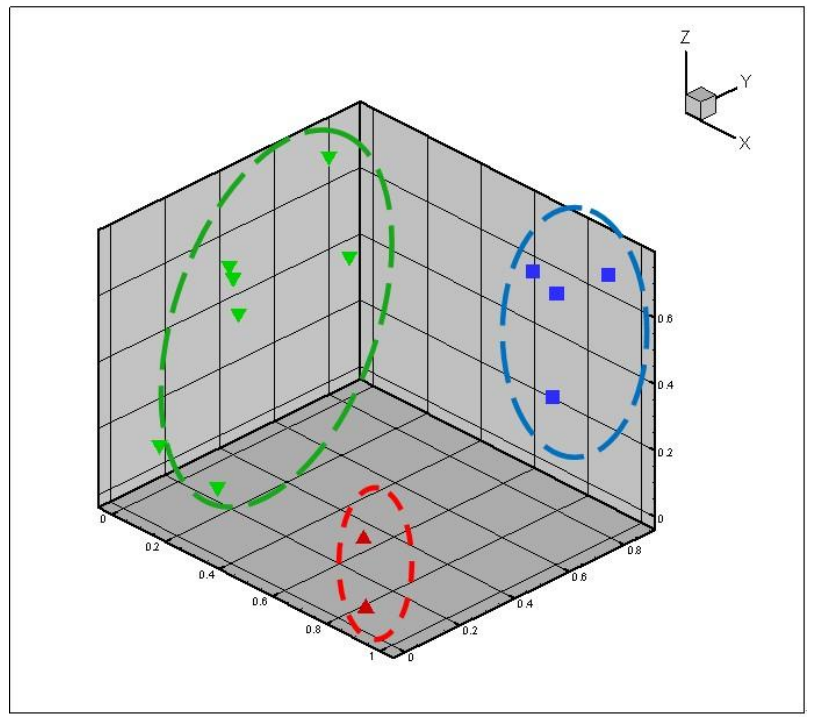

Figure 6. Scattered sample points

\section{B. Summary of simulation results based on BP network}

After superfluous data are ruled out, data of 9 dimensions remain, while 4 dimensions are used for input and other 5 dimensions are output. And qualitative mapping relations are got and shown as follow:

1) If luminous flux is set $10001 \mathrm{~m}$ and illuminant color temperature is set $6000 \mathrm{~K}$, brightness, luminous intensity, contrast, color $\mathrm{x}$ and color $\mathrm{y}$ are the function of material absorption, color coordinate $\mathrm{x}$ and color coordinate $\mathrm{y}$.

2) If material properties are given and illuminant color temperature is set $6000 \mathrm{~K}$ as well, brightness and luminous intensity are monodrome function of luminous flux.

Constrain condition: Material transmissivity is 0 . Luminous flux is $1000 \mathrm{~lm}$. Illuminant color temperature is $6000 \mathrm{~K}$.

Establish the corresponding relationship between material absorption, color coordinate $\mathrm{x}$, color coordinate $\mathrm{y}$, and color $\mathrm{x}$, color y by Matlab neural network toolbox. Total samples are 15 . 12 of them are training samples, while the others are testing samples. BP network adopts 3-layer structure, 4 input neuron, 2 output neuron and 2000 training steps. After training, the error salvage value curve is shown in Figure 7. The training sample and testing sample are shown in Figure 8(a) and Figure 8(b). The main error of testing sample is $-0.19 \%$. 


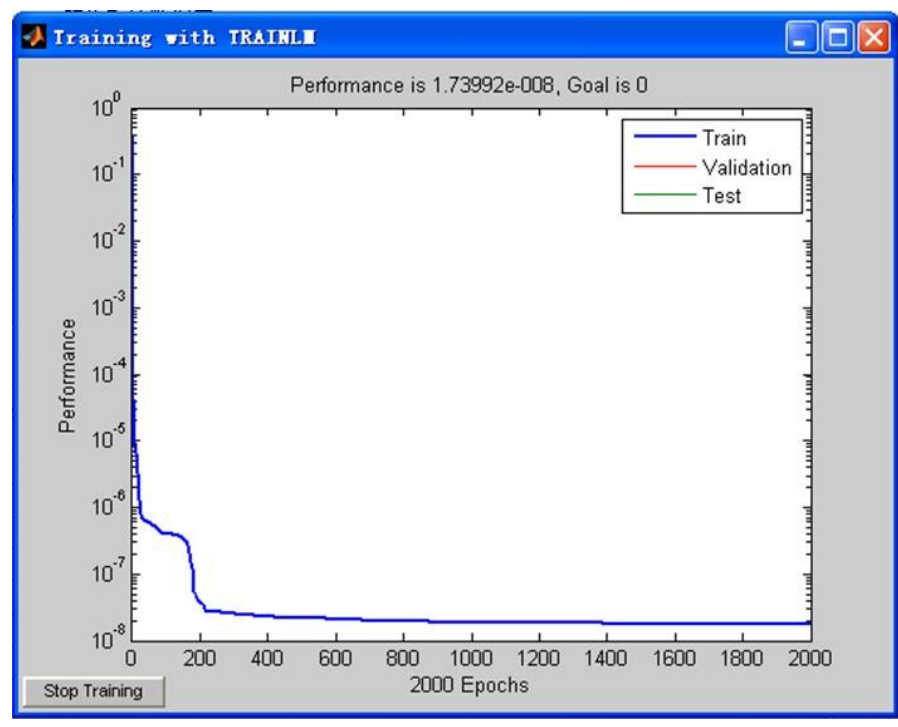

Figure 7. Error salvage value curve

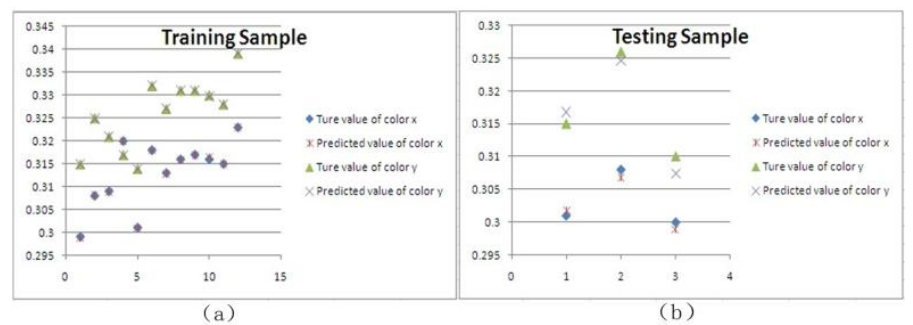

Figure 8. (a) Training Sample and (b) Testing Sample

Establish the corresponding relationship between absorption, color coordinate $\mathrm{x}$, color coordinate $\mathrm{y}$, and contrast. Then the training results of color $\mathrm{x}$ and color $\mathrm{y}$ are shown in Table $\mathrm{V}$, as well as training results of contrast in Table VI. Although average error is $0.01 \%$, maximum error is about $5 \%$. The main reasons are the number of samples is relatively a little fewer and the corresponding relationship is uncertain, which will be investigated further. The corresponding relationship between material absorption, color coordinate $\mathrm{x}$, color coordinate $\mathrm{y}$, and brightness, luminous intensity is obtained through Matlab neural network. The result doesn't perform well, which needs further study.

If material properties are given and illuminant color temperature is set $6000 \mathrm{~K}$ as well, brightness and luminous intensity are almost direct proportional to luminous flux. The following figures are data researches of two materials. Approximating by a linear function with intercept of zero, square of linearity $\mathrm{R}$ is 0.9997 , which is in the range of allowable error. The relationship of brightness and luminous intensity of different material is shown in (a)-(d) of Figure 9.
Obviously, brightness is liner with luminous intensity. Without building neural network, brightness and luminous intensity of random luminous flux can be calculated according to brightness and luminous intensity of different material with $10001 \mathrm{~m}$ aforementioned.

TABLE V.

\begin{tabular}{cccccc}
\hline Color x & Color y & $\begin{array}{c}\text { Training } \\
\text { Results of } \\
\text { Color x }\end{array}$ & $\begin{array}{c}\text { Training } \\
\text { Results of } \\
\text { Color y }\end{array}$ & Error x & Error y \\
\hline 0.301 & 0.315 & 0.302 & 0.317 & $0.27 \%$ & $0.57 \%$ \\
0.308 & 0.326 & 0.307 & 0.325 & $-0.39 \%$ & $-0.43 \%$ \\
0.3 & 0.31 & 0.299 & 0.308 & $-0.37 \%$ & $-0.81 \%$ \\
0.299 & 0.315 & 0.299 & 0.315 & $-0.03 \%$ & $0.00 \%$ \\
0.308 & 0.325 & 0.308 & 0.325 & $0.06 \%$ & $-0.03 \%$ \\
0.309 & 0.321 & 0.309 & 0.321 & $-0.03 \%$ & $0.00 \%$ \\
0.32 & 0.317 & 0.320 & 0.317 & $0.00 \%$ & $0.00 \%$ \\
0.301 & 0.314 & 0.301 & 0.314 & $0.00 \%$ & $0.00 \%$ \\
0.318 & 0.332 & 0.318 & 0.332 & $-0.03 \%$ & $0.03 \%$ \\
0.313 & 0.327 & 0.313 & 0.327 & $-0.06 \%$ & $0.03 \%$ \\
0.316 & 0.331 & 0.316 & 0.331 & $-0.06 \%$ & $0.03 \%$ \\
0.317 & 0.331 & 0.317 & 0.331 & $-0.03 \%$ & $0.03 \%$ \\
0.316 & 0.33 & 0.316 & 0.330 & $0.13 \%$ & $-0.06 \%$ \\
0.315 & 0.328 & 0.315 & 0.328 & $0.00 \%$ & $0.00 \%$ \\
0.323 & 0.339 & 0.323 & 0.339 & $0.00 \%$ & $0.00 \%$ \\
\hline
\end{tabular}

TABLE VI.

\begin{tabular}{ccc}
\hline Contrast & $\begin{array}{c}\text { Training } \\
\text { Results of } \\
\text { Contrast }\end{array}$ & Error \\
\hline 0.6554 & 0.625 & $-4.64 \%$ \\
0.3478 & 0.345 & $-0.81 \%$ \\
0.6731 & 0.7077 & $5.14 \%$ \\
0.4792 & 0.4791 & $-0.02 \%$ \\
0.3543 & 0.3718 & $4.94 \%$ \\
0.3422 & 0.3435 & $0.38 \%$ \\
0.6162 & 0.6167 & $0.08 \%$ \\
0.3615 & 0.3498 & $-3.24 \%$ \\
0.7294 & 0.7256 & $-0.52 \%$ \\
0.4378 & 0.4295 & $-1.90 \%$ \\
0.6515 & 0.6515 & $0.00 \%$ \\
0.3416 & 0.3416 & $0.00 \%$ \\
0.6861 & 0.6907 & $0.67 \%$ \\
0.3522 & 0.3522 & $0.00 \%$ \\
0.3633 & 0.3633 & $0.00 \%$ \\
\hline
\end{tabular}



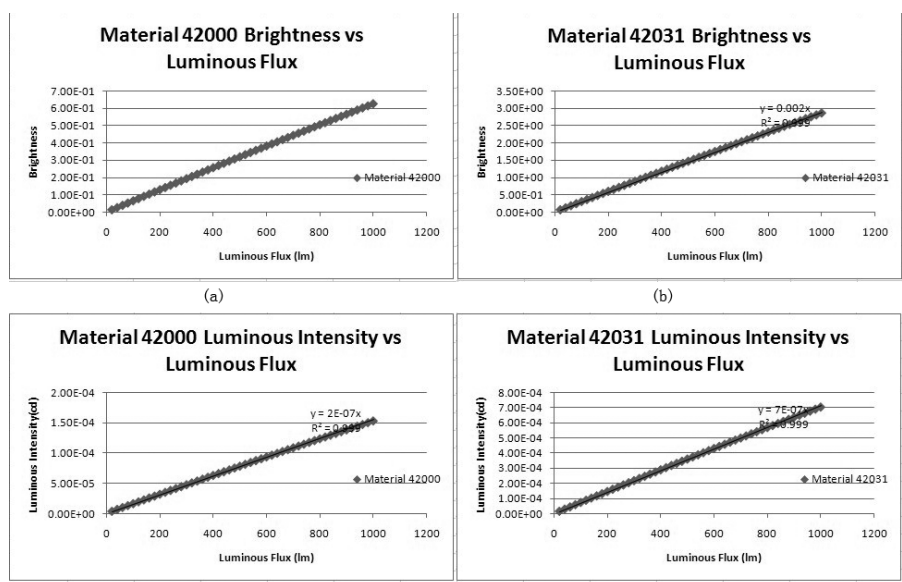

(c)

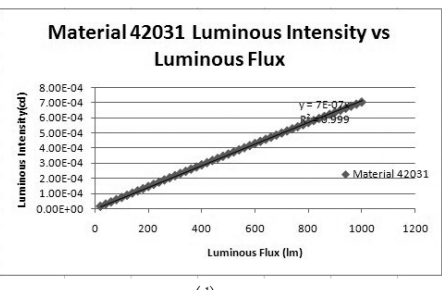

(d)

Figure 9.

\section{CONClusions}

This paper present a system used for multivariable coupling by ANNs method. The method proves to be usefull and effective. SOM network is used for selecting different materials variables while BP network is used for non-linear fit. Approximate relationship between material variables and photometric variables established, so that there's corresponding output for arbitrary input within the approximate relationship. In this way, large amount of data would be obtained without experiment.

In this paper, although the case has 9 dimensions, it can also be applied into more dimensions. The degree of accuracy is depended on the scale of database.

\section{ACKNOWLEDGMENT}

The work is sponsored by National Basic Research Program (“973” Program) of China, Issue Number 2010CB734106.

\section{REFERENCES}

[1] J. B. Ding. "Synthetic Fuzzy Evaluation of the Quality of Light Conditions of the Human2Machine System", Journal of Xuzhou Institute of Architectureal Technology, 2(2), 11-18 (2002).

[2] L. Z. Ge and F. P. Hu, "The Ergonomics Study on Multi-element Visual Display” Journal of Developments in psychology, 9(3), 201-204 (2001).

[3] D. Q. Zhang, Z. J. Zhang and H. Z. Yang, "VDT interface color vision work efficiency: Tonal factors on the visual performance impact," Psychological Science, 31(2), 328-331 (2008).

[4] Liqun Han. "Artificial neural network course. Beijing University of Posts and Telecommunications Press, (2006).

[5] M. Hagan. Neural Network Design. China Machine Press, (2002).

[6] M. A. L. Nicolelis and S. Ribeiro "Seeking the neural code", Scientific American, 295(6), 70-77 (2006).

[7] T. Kohonen, “The Self-Organizing Map", IEEE, 78(9), 1464-1484, (1990).

[8] X. Wen, chap. 10 in Matlab neural network simulation and application, pp. 250-300, Science Press, Beijing (2003). 